OPEN ACCESS

Edited by:

Bing Wang,

Anhui University of Technology, China

Reviewed by:

Quan Zou,

University of Electronic Science and Technology of China, China

Lei Wang,

Xiangtan University, China

*Correspondence:

Wen Zhu

syzhuwen@163.com

Specialty section:

This article was submitted to

Preclinical Cell and Gene Therapy,

a section of the journal

Frontiers in Bioengineering and

Biotechnology

Received: 16 September 2020

Accepted: 26 October 2020

Published: 24 November 2020

Citation:

Liang $X$, Zhu W, Liao B, Wang B,

Yang J, Mo X and Li R (2020) A

Machine Learning Approach

for Tracing Tumor Original Sites With

Gene Expression Profiles.

Front. Bioeng. Biotechnol. 8:607126.

doi: 10.3389/fbioe.2020.607126

\section{A Machine Learning Approach for Tracing Tumor Original Sites With Gene Expression Profiles}

\author{
Xin Liang ${ }^{1,2,3}$, Wen Zhu ${ }^{1,2,3 *}$, Bo Liao ${ }^{1,2,3}$, Bo Wang ${ }^{4,5}$, Jialiang Yang ${ }^{4,5}$, Xiaofei Mo ${ }^{4,5}$ and \\ Ruixi $L i^{1,2,3}$ \\ 'Key Laboratory of Computational Science and Application of Hainan Province, Haikou, China, ${ }^{2}$ Key Laboratory of Data \\ Science and Intelligence Education, Ministry of Education, Hainan Normal University, Haikou, China, ${ }^{3}$ School of Mathematics \\ and Statistics, Hainan Normal University, Haikou, China, ${ }^{4}$ Qingdao Geneis Institute of Big Data Mining and Precision \\ Medicine, Qingdao, China, ${ }^{5}$ Geneis (Beijing) Co., Ltd., Beijing, China
}

Some carcinomas show that one or more metastatic sites appear with unknown origins. The identification of primary or metastatic tumor tissues is crucial for physicians to develop precise treatment plans for patients. With unknown primary origin sites, it is challenging to design specific plans for patients. Usually, those patients receive broadspectrum chemotherapy, while still having poor prognosis though. Machine learning has been widely used and already achieved significant advantages in clinical practices. In this study, we classify and predict a large number of tumor samples with uncertain origins by applying the random forest and Naive Bayesian algorithms. We use the precision, recall, and other measurements to evaluate the performance of our approach. The results have showed that the prediction accuracy of this method was 90.4 for 7,713 samples. The accuracy was $80 \%$ for 20 metastatic tumors samples. In addition, the 10-fold cross-validation is used to evaluate the accuracy of classification, which reaches $91 \%$.

Keywords: the ability of tissue tracing, random forest, naive Bayes, machine learning, uncertain origins

\section{INTRODUCTION}

Tumors can develop in any part of body, and some tumors even can metastasize to other parts of the body from their primary sites after developing at a certain point. In general, the occurrence of tumors at primary sites and their metastatic sites could be found deferentially, and the primary origins of metastatic cancers can be identified within a short amount of time by clinical assessments (Chen and Chen, 2001). Histological and imaging techniques are mostly employed to identify the origin of metastatic tumors. However, in some cancer patients, physicians cannot find the primary origin of tumors even after comprehensive examinations and assessment studies of patients with standard methods. These tumors are called carcinomas with unknown primary (CUP). According to statistical data, there are approximately 150,000 new cases of CUPs annually in the United States and Europe, and the numbers are still increasing though. Currently, approximately one third of cancer patients would develop metastasis after initial diagnosis and/or post-operation treatment. In many of those patients, it is relatively difficult for physicians to identify the primary origins of the metastatic cancers (Oien, 2009; Pavlidis and Pentheroudakis, 2012). To our knowledge, $2-4 \%$ of CUPs (Susman et al., 2012) account for all metastatic cancer. Even through autopsy, the 
primary origin of CUPs is uncertain (Myung et al., 2001; Petrushev et al., 2011). Because of limited treatment plan for CUP patients, the treatment efficacy is often unpredictable, and those patients usually have poor prognosis (Sun and Zhang, 2006; Gupta et al., 2007; Carmeliet and Jain, 2011; Petrakis et al., 2013). The immunohistochemistry assay is usually considered to be a diagnostic method for CUP patients. However, it is timeconsuming and subjective. Moreover, the diagnostic accuracy is around by $30 \%$ for CUP patients, which is not reliable to design a personalized treatment plan for CUP patients. Currently, most CUP patients received radiological therapy (Stoyianni et al., 2011) or broad-spectrum chemotherapy. However, these treatments are not effective and with intolerable complications, and the prognosis is relatively poor as well. Therefore, it is urgent to develop effective clinical intervention for CUP patients (Guntinas-Lichius et al., 2006; Pavlidis and Fizazi, 2009; Hainsworth and Greco, 2014). Nowadays, identifying the primary origin of malignant tumors is critical for designing a treatment plan in clinical practices.

The targeted therapy (Tsao et al., 2005; Hudis, 2007; Miller et al., 2007; Varadhachary et al., 2008; Anderson and Weiss, 2010; Boscolo-Rizzo et al., 2015) can be used for tumors after accurately identifying the primary origin, which could greatly improve the survivals. It has been proven in the Minnie Pearl Cancer Research Network Study (Pavlidis and Pentheroudakis, 2010; Molina et al., 2012). Immunohistochemically, the marker has also been an important instrument for identifying the primary origin of cancerous tissues (Monzon et al., 2009; MacReady, 2010; Massard et al., 2011; Hashimoto et al., 2012; Oien and Dennis, 2012; Kim et al., 2013; Tang et al., 2018). Furthermore, a diagnostic method has been proposed to predict the primary origin of malignant cancers by comparing the gene expression profiles from the primary origin and the metastasis tissue (Hoadley et al., 2014). Many researchers have systematically compared the characteristics of gene expression profile across different cancers (Joyce and Pollard, 2009). Therefore, it is feasible to compare the differential gene expression to predict the primary origin of malignant cancer. There are two commercial products approved by FDA, which are Tissue of Origin (TOO) and CancerTYPE ID. Both of them are developed on the basis of differential gene expressions to predict primary origins.

TOO is a product of array-based gene expression profiles. TOO can identify 2,000 genes and 15 types of tumors, including thyroid cancer, breast cancer, non-small cell lung cancer, pancreatic cancer, gastric cancer, colorectal cancer, liver cancer, bladder cancer, kidney cancer, non-Hodgkin's lymphoma, melanoma, ovarian cancer, sarcoma, testicular germ cell tumor, and prostate cancer. The advantage of this product is that it prevents the subjective bias. It can objectively identify the primary origin of cancers no matter which is well-differentiated or not. However, TOO is time-consuming, which is not feasible for clinical practices (Brugarolas, 2007; Economopoulou et al., 2015).

CancerTYPE ID is a product that uses cancer samples based on RT-PCR data. In the study (Marquard et al., 2015), 578 labeled samples covering 39 tumor types were included in datasets for tracing origins. The results showed that there was no significant difference in the accuracy of predictions of cancer with primary or metastatic tumors. Secondly, RT-PCR was used to evaluate the 92-gene ( $\mathrm{Ma}$ et al., 2005) expression of cancer cells from patients and then compared with labeled 50 tumors from databases to predict the primary origin of metastatic tumors and their subtypes (Pappa et al., 2006). CancerTYPE ID has been able to compare gene expression profiles from tumor samples to reference database with more than 2,000 labeled tumors, therefore identifying the most accurate results. However, CancerTYPE ID does not have the relatively good accuracy for pancreatic cancer, colorectal cancer, and gastroesophageal cancer.

Though the above two products have good performance for some types of cancers, two products are costly with up to $\$ 3000$ \$4000 (Pillai et al., 2011; Oien and Dennis, 2012; Economopoulou et al., 2015), and the accuracy is limited to other types of cancer as well. In order to facilitate the low-cost and high-efficiency product, our study aimed to use RNA-seq data, which are extracted from TCGA database, combining with random forest and naïve Bayes algorithms to develop a computational model.

\section{RESULTS}

Firstly, data were downloaded from TCGA and GEO. Secondly, after data preprocessing for raw data, genes were selected by the random forest algorithm with 10 -fold cross-validation. Finally, the naive Bayes classifier was used to classify the 20 kinds of tumors, and the output of the model was shown as the evaluation index. The detailed step is shown in Figure 1.

\section{Data Preparation}

A total of 7,715 RNA-seq samples that covered 21 cancers and excluded metastatic cancers were extracted from TCGA. In the process of data preparation, we eliminated two samples due to the lack of clinical data. Therefore, the remaining 7,713 samples were used as either the training dataset or the validation dataset for the classification. Furthermore, the expression spectrum matrix of 7,633 samples was constructed. Each sample contained 20,501 genes. In this paper, 372 samples from metastatic cancers were selected as the test dataset, of which 352 samples belong to Skin Cutaneous Melanoma (SKCM). The ratio of SKCM was much higher than other types of metastatic cancers, and we excluded SKCM data from our selected data in order to reduce the possible effects on the results. The detailed information of selected data is shown in Table 1.

For the independent validation dataset, 48 samples are obtained from GEO and processed according to the description in section "Materials and Methods" and then used for the trained naive Bayesian model to make the prediction. The detailed information of selected data is shown in Table 2.

\section{Gene Selection by Random Forests}

Under the common condition, we use relatively low-cost panels but also include sufficient genes to determine the level of specific gene expression. However, the coverage of gene numbers would be significantly affected by the cost of panel. In order to reduce 


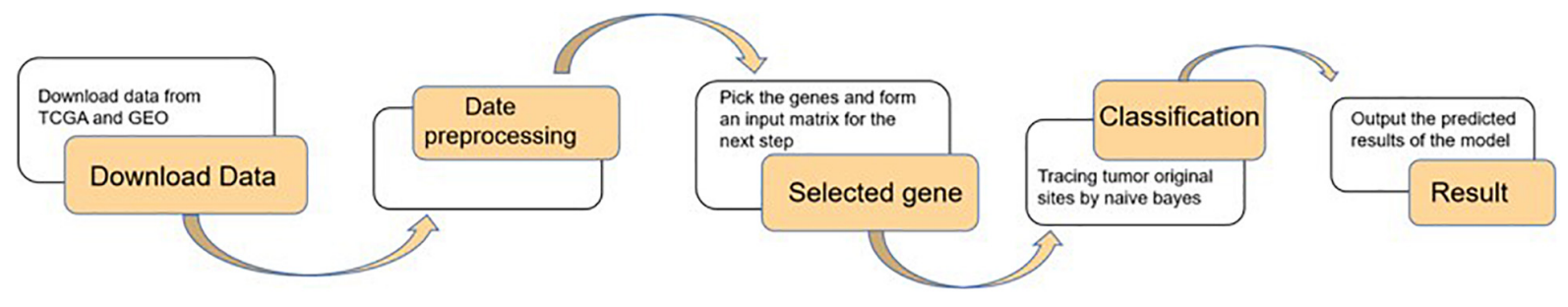

FIGURE 1 | Flow chart of the article.

the cost of panels as well as improve the accuracy of tracing ability. Random forest algorithm was employed widely in the bioinformatics researches (Lv et al., 2019, 2020; Ru et al., 2019).

TABLE 1 | Detailed information of data covering 21 cancers downloaded from TGCA.

\begin{tabular}{lccccc}
\hline Cancer & $\begin{array}{c}\text { Total } \\
\text { samples }\end{array}$ & $\begin{array}{c}\text { Samples } \\
\text { from } \\
\text { women }\end{array}$ & $\begin{array}{c}\text { Samples } \\
\text { from men }\end{array}$ & $\begin{array}{c}\text { Percentage } \\
\text { (\%) }\end{array}$ & Note \\
\hline BLCA & 301 & 80 & 221 & 3.9 & \\
BRCA & 1,056 & 1,044 & 11 & 13.7 & 1 person has no \\
& & & & & clinical information \\
CESC & 258 & 258 & 0 & 3.3 & \\
COAD & 451 & 215 & 236 & 5.8 & \\
GBM & 153 & 53 & 100 & 2.0 & \\
HNSC & 480 & 128 & 352 & 6.2 & \\
KIRC & 526 & 184 & 342 & 6.8 & \\
KIRP & 222 & 63 & 159 & 2.9 & \\
LAML & 173 & 80 & 93 & 2.2 & \\
LGG & 439 & 192 & 247 & 5.7 & \\
LIHC & 294 & 99 & 195 & 3.8 & \\
LUAD & 486 & 262 & 224 & 6.3 & \\
LUSC & 428 & 109 & 319 & 5.5 & \\
OV & 261 & 261 & 0 & 3.4 & \\
PAAD & 142 & 64 & 78 & 1.8 & \\
PRAD & 379 & 0 & 379 & 4.9 & \\
READ & 153 & 70 & 82 & 2.0 & 1 person has no \\
& & & & & clinical information \\
SKCM & 80 & 34 & 46 & 1.0 & \\
STAD & 415 & 147 & 268 & 5.4 & \\
THCA & 500 & 367 & 133 & 6.5 & \\
UCEC & 516 & 516 & 0 & 6.7 & \\
Total & 7,713 & 4,226 & 3,485 & 99.8 & \\
& & & & & \\
\end{tabular}

TABLE 2 | Detailed information of data covering five cancers downloaded from GEO.

\begin{tabular}{lcc}
\hline Cancer & Total samples & Percentage (\%) \\
\hline LIHC & 9 & 18.75 \\
UCEC & 6 & 12.5 \\
THCA & 8 & 16.67 \\
BLCA & 11 & 22.92 \\
PAAD & 14 & 29.17 \\
Total & 48 & 99.98
\end{tabular}

In this study, the random forest algorithm was applied to select the features of the primary origin tumor samples, and a matrix of $M^{*} N$ was formed, with $M$ representing the number of samples and $\mathrm{N}$ representing the numbers of genes, and all samples were labeled with the type of each cancer. The expression profile was divided into 20 types of cancer, and the combination of five genes could be used to classify this problem (Ashburner et al., 2000). The Gini average impurity method of random forest was used as the standard to evaluate the importance of genes. The importance score of genes was obtained, and the genes were sequenced according to the score. We conducted many experiments, and the precision was the highest when 2,300 genes were obtained. The experimental results are shown in Figure 2. Our method takes five steps and increases $\mathrm{N}$ up to 2,300.

Based on the above analysis, genes with high scores were selected as the features, and 2,300 genes were extracted from each sample. Because some genes were not in the GEO database, we deleted these genes and got 2,284 genes. A 7,633*2,284 matrix was constructed as the input matrix for cancer classification.

\section{Classification Based on Naive Bayes}

Since Naive Bayes is relatively consistent for classification, this study used Naive Bayes as a classifier for genomic combination. In this study, we chose $75 \%$ of the dataset for training, and the remaining $25 \%$ was chosen for validation by using our model. The algorithm used gene expression as the feature for training and predicting the labeled cancer. After the training, the model achieved the accuracy of $91 \%$ in predicting the origin of the cancer. In order to validate the accuracy of classification of model for metastatic tumors, 20 metastatic tumors with known primary origin sites were applied to the model. 7,633*2,284 was used as the input matrix for classification and applied to the naive Bayesian classification model to obtain the specific prediction results of specific cancer types with a prediction accuracy of $80 \%$ for metastatic cancer types, as shown in Figure 3.

In addition, ClueGO was used to identify gene ontology and enrichment analysis for selected genes. Due to the large number of 2,284 genes, we selected the top 100 genes with the highest score for analysis. The statistical significance level is set as the $p$-value of 0.001 . The results of enrichment analysis are shown in Figure 4.

The enrichment results in Figure 4 show that the genes are significantly enriched in cellular metabolism, especially 


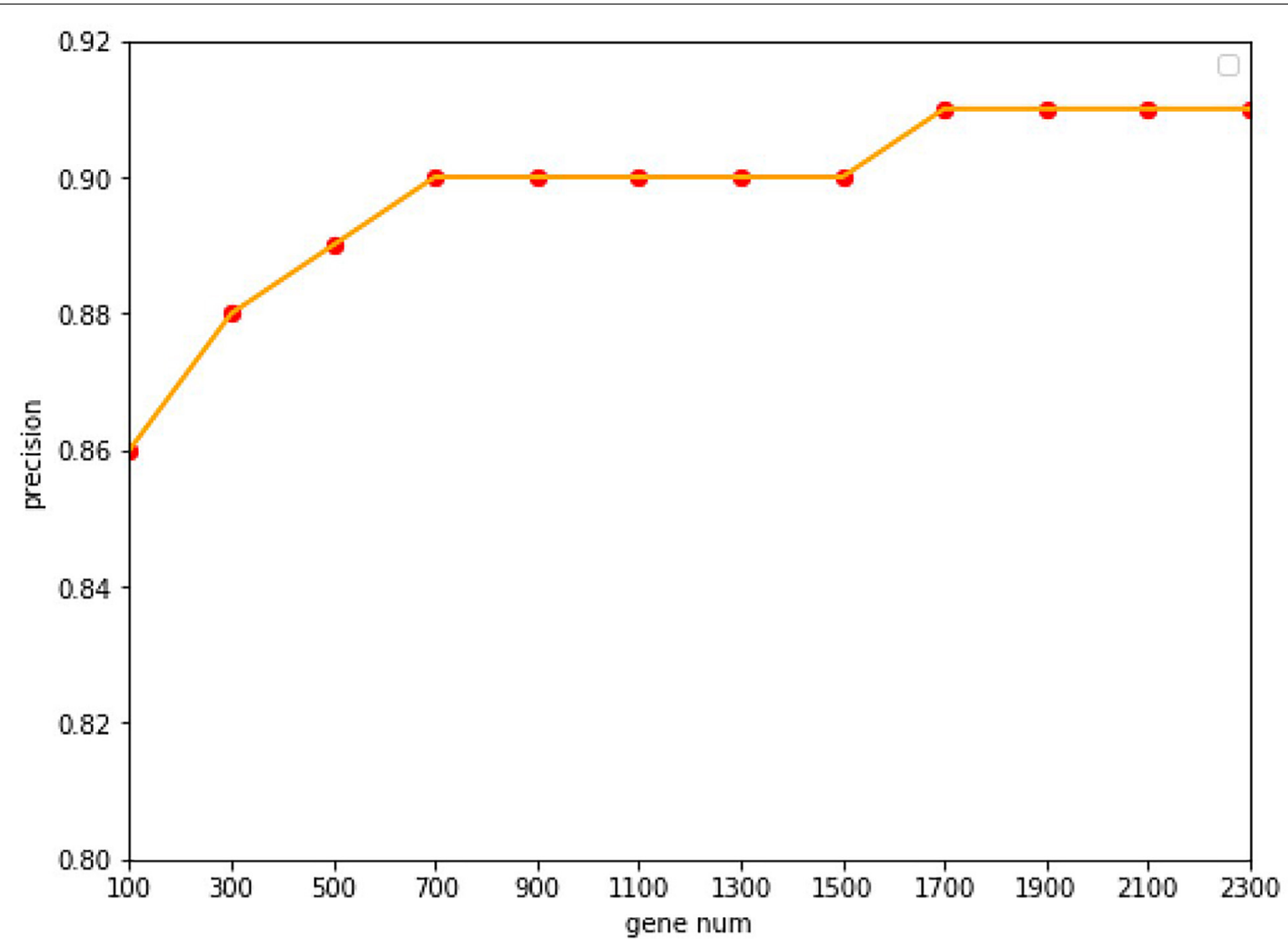

FIGURE 2 | The accuracy with the different of number genes. With a 10-fold cross-validation accuracy, the value of the accuracy is increasing up to 1,700 genes, after which it keeps stable with the value of $91.07 \%$.

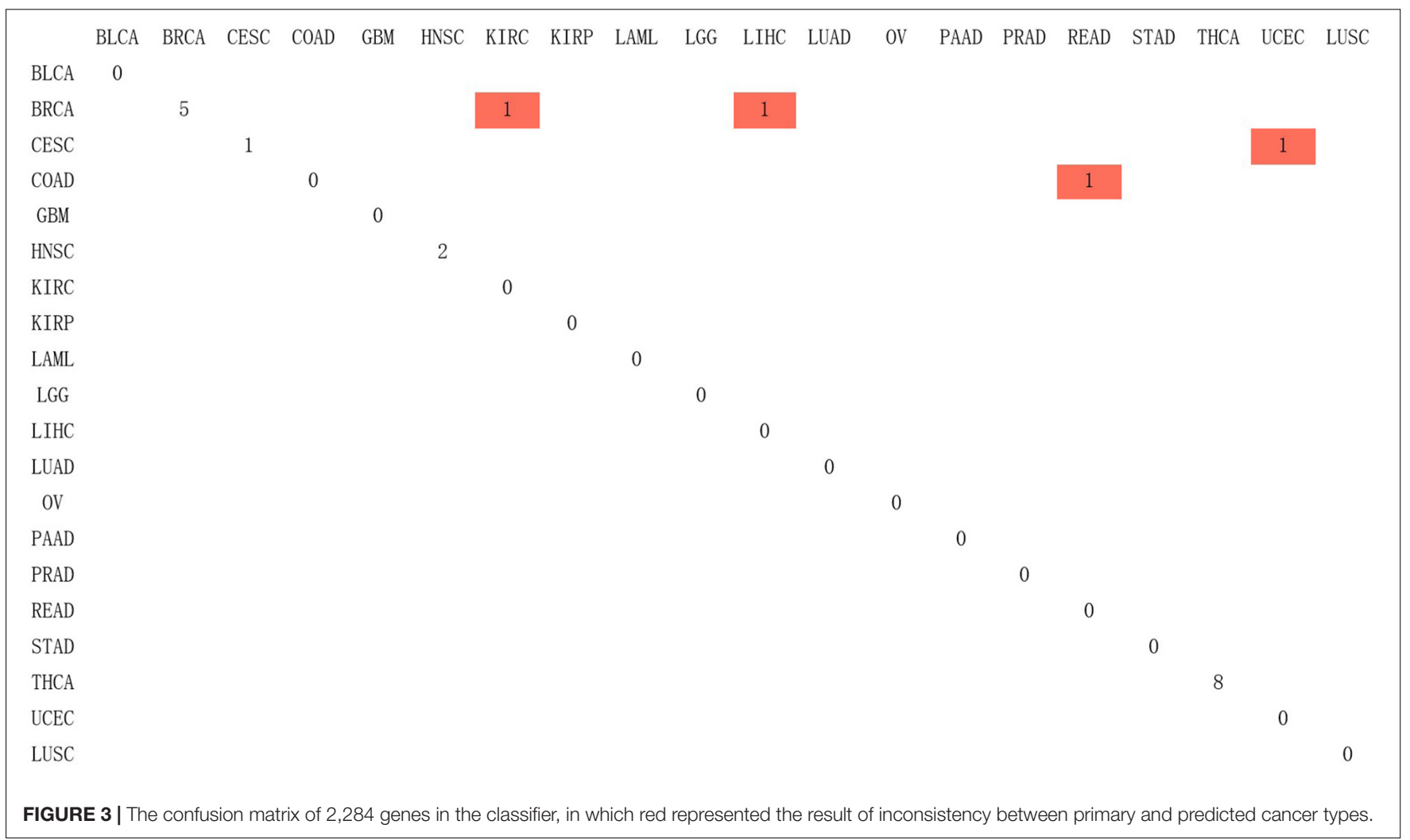




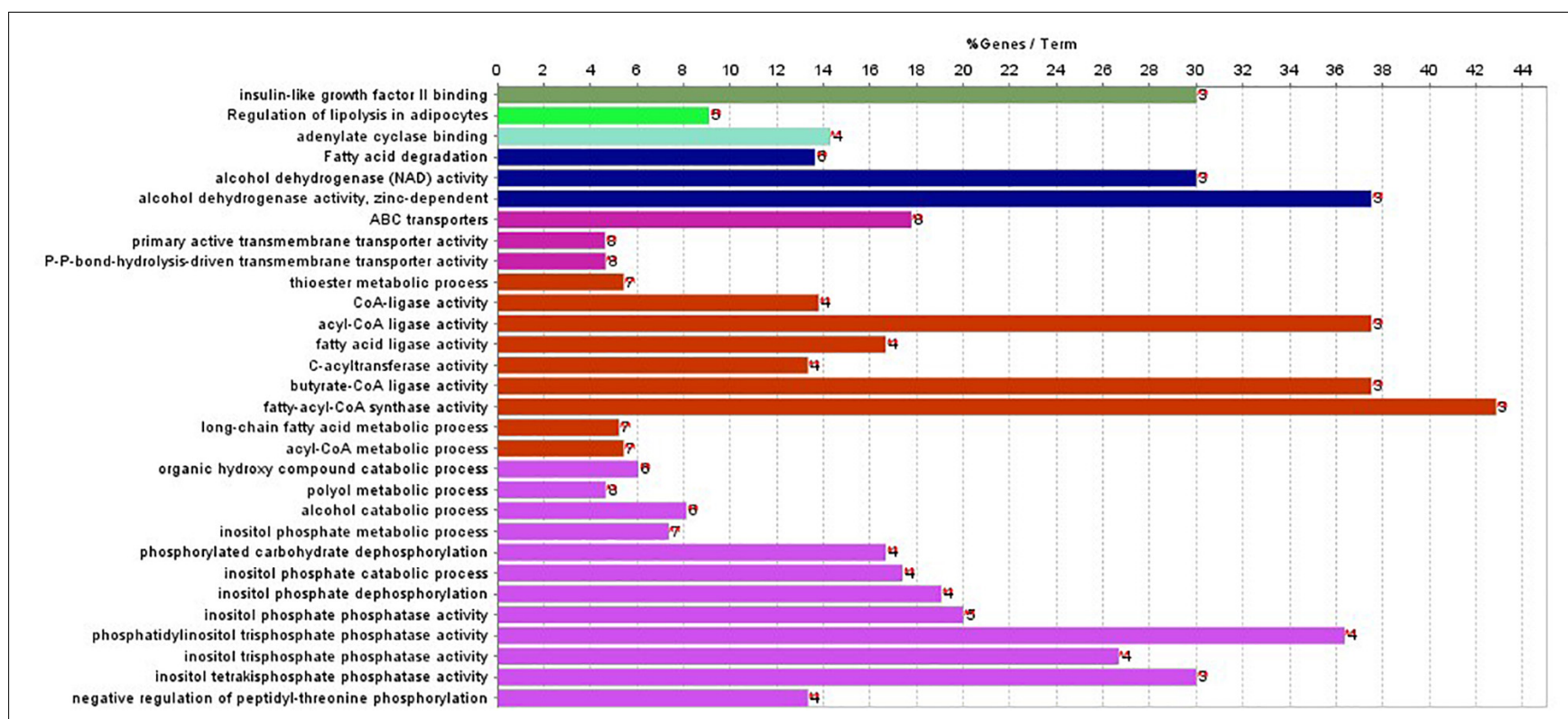

FIGURE 4 | Gene enriched in biological process, cellular component, and molecular function were drawn for first 100-gene set by ClueGO.

lipid metabolism. In addition, some genes are enriched in acetyl-CoA cycle, alcohol dehydrogenase NAD activity, etc. Almost all genes are enriched in lipid metabolism, which provides cellular energy for all cellular activity. Moreover, genes are also enriched through peroxisome proliferator-activated receptor (PPAR) signaling pathway. PPARs are nuclear hormone receptors activated by fatty acids and their derivatives and belong to ligand-activated receptors in the nuclear hormone receptor family. The PPAR signaling pathway plays a role in clearance of circulating lipid and promotes lipid oxidation and cell proliferation. The PPAR transcriptional activity can be regulated by non-gene crosstalks with phosphatases and kinases, including ERK1/2, p38-MAPK, PKC, and AMPK. The upregulated PPAR signaling pathway would lead to dysfunctional metabolic homeostasis and inflammatory response, ROS accumulation, as well as carcinogenesis across almost every tumor.

In order to further differentiate those 100 genes, the following heat map was drawn to further reveal the gene expression level in each cancer type.

The analysis shown in Figure 5 reveals that there are expression differences of the first 100 genes in different cancers. Each small block represents a gene, and the color represents the size of gene expression. The higher level of the expression is represented with the darker color (red indicates upregulated and green indicates downregulated). The bottom horizontal line represents a different gene, while the vertical line on the right represents a different cancer.

\section{Independent Verification}

For independent tests, the model with the previous training parameters was tested on the dataset in GEO, and the probability of each sample being accurately assigned to each category was given, with an overall accuracy of $75 \%$. The specific results are shown in Figure 6.

\section{Performance Assessment}

For the evaluation of classification performance, this study used the 10 -fold cross-validation for the algorithm with the feature in each gene set. To be specific, the samples were randomly divided into 10 subsets; 1 of 10 subsets was selected as the test set at one time, and the other 9 was merging to 1 training set. The accuracy of cross-validation is $90 \%$, which indicated that the algorithm achieved a good performance. The precision, recalls, and f1 scores were used to evaluate the significance of the model as well. The detailed results are shown in Figure 7.

The comparison among results of the $k$-nearest neighbor $(k=5)$, decision tree, and Naive Bayesian to classify 20 cancers is shown in Figure 8.

\section{MATERIALS AND METHODS}

\section{Data Preparation}

The TCGA RNA-seq and array data were downloaded from the ICGC Data Portal ${ }^{1}$. Each sample and each gene from each cancer type table were extracted to generate a matrix of $\mathrm{M}^{*} \mathrm{~N}$, where $\mathrm{M}$ is the number of samples, $\mathrm{N}$ is the number of genes, and all the samples were labeled by cancer types. All primary tumors were divided into training sets and metastatic tumors were divided into test sets.

For the independent set, 48 samples from 5 known cancer origin sites were downloaded from Gene Expression Omnibus (GEO). These tumors belong to GSE10907, GSE11222,

\footnotetext{
${ }^{1}$ https://dcc.icgc.org/releases/release_26/
} 

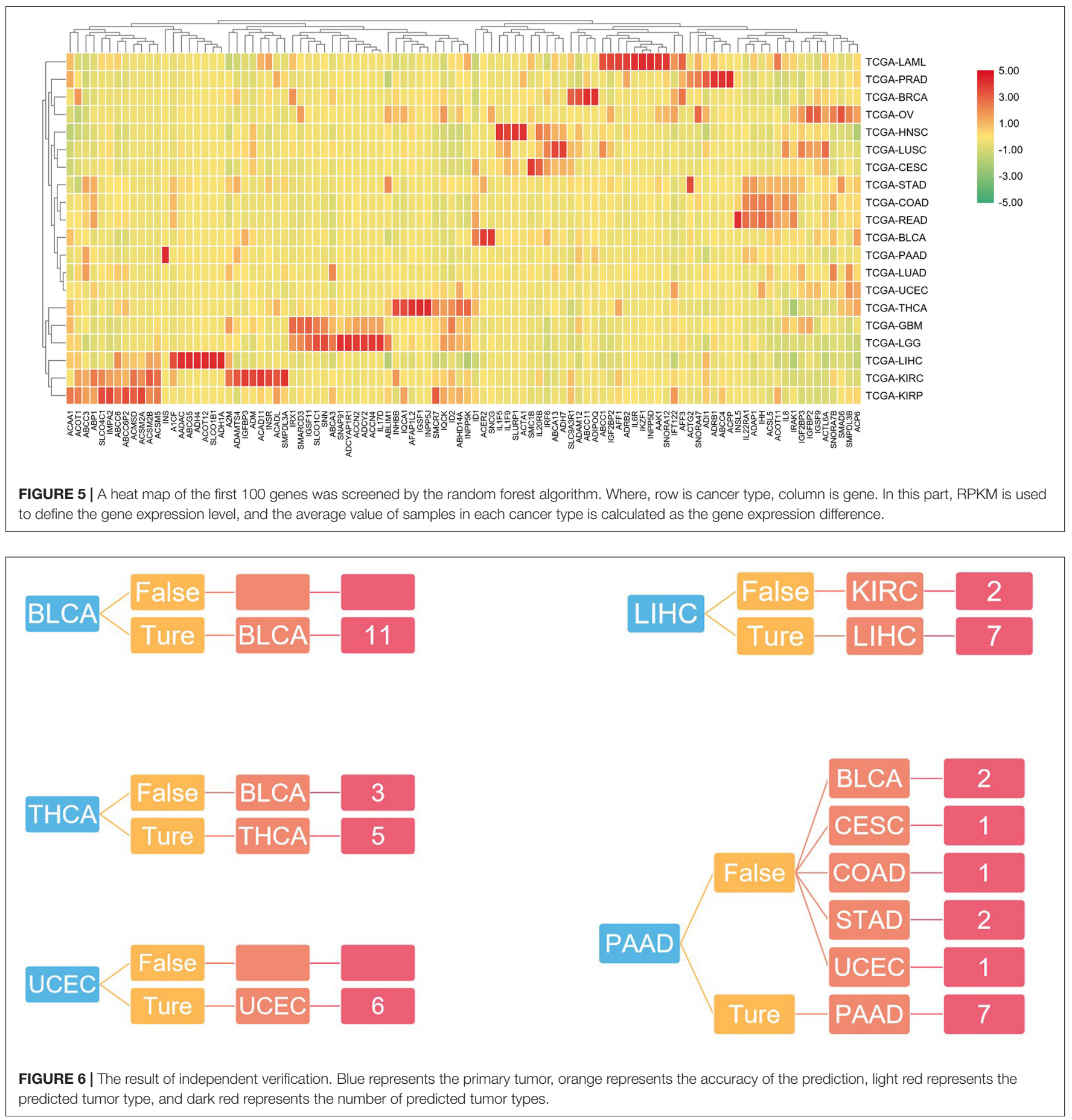

GSE5608, GSE8352, GSE4895, GSE8912, GSE7966, and GSE12281. In addition, these 5 cancers belong to the 20 cancer types in this paper.

\section{Gene Selection}

In order to reduce the cost of gene number determined by gene panel, in this study, random forest algorithm was applied to select genes. The Gini average impurity in random forest was used as the criterion to estimate the importance of genes. The random forest is composed of several decision trees, which are binary decision trees. Each node in the decision tree is a condition on a single gene. As a result, we can achieve the goal by splitting the dataset into two datasets; therefore, a similar expression level can be classified in the same dataset. For random forest, the average reduction of each feature impurity can be calculated. In addition, the importance score of genes can be calculated and sorted according to the score. GI stands for Gini, S stands for importance score, $G=\left\{g_{1}, g_{2}, . . g_{n}\right\}$ stands for feature, and $C$ stands 


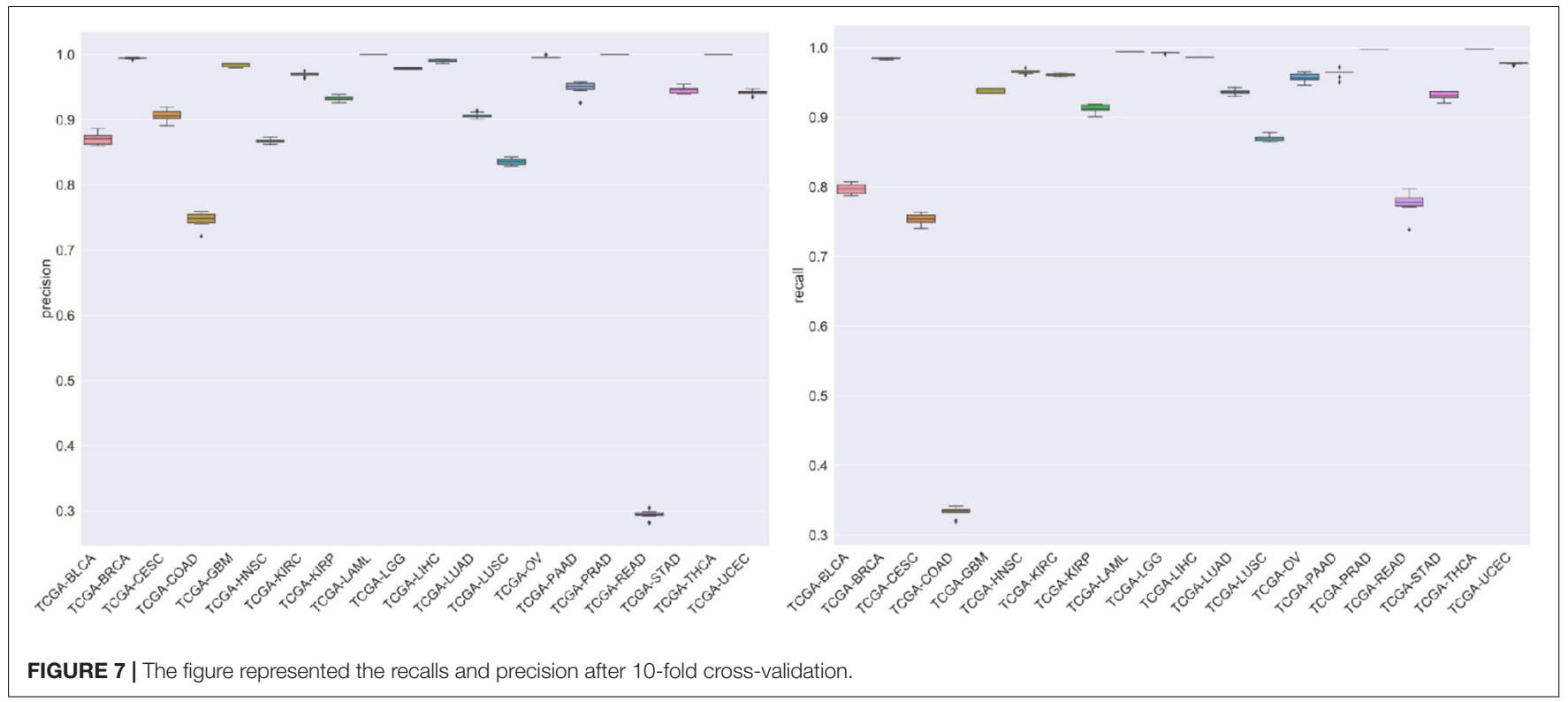

\begin{tabular}{|c|c|c|c|c|c|c|c|c|c|c|c|c|}
\hline \multirow[b]{2}{*}{ cancer type } & \multicolumn{4}{|c|}{ KNN } & \multicolumn{4}{|c|}{ decision tree } & \multicolumn{4}{|c|}{ naive Bayesian } \\
\hline & precision & recall & f1-score & support & precision & recall & f1-score & support & precision & recall & f1-score & support \\
\hline TCGA-BLCA & 0.73 & 0.7 & 0.71 & 80 & 0.76 & 0.7 & 0.73 & 80 & 0.85 & 0.79 & 0.8 & 80 \\
\hline TCGA-BRCA & 0.95 & 0.97 & 0.96 & 274 & 0.93 & 0.94 & 0.93 & 274 & 0.99 & 0.98 & 0.99 & 274 \\
\hline TCGA-CESC & 0.53 & 0.51 & 0.51 & 59 & 0.56 & 0.59 & 0.57 & 59 & 0.88 & 0.71 & 0.79 & 59 \\
\hline TCGA-COAD & 0.7 & 0.84 & 0.76 & 99 & 0.69 & 0.7 & 0.69 & 99 & 0.73 & 0.36 & 0.49 & 99 \\
\hline TCGA-GBM & 0.84 & 0.66 & 0.74 & 32 & 0.9 & 0.88 & 0.89 & 32 & 0.97 & 0.94 & 0.95 & 32 \\
\hline TCGA-HNSC & 0.82 & 0.85 & 0.83 & 117 & 0.95 & 0.96 & 0.95 & 117 & 0.84 & 0.94 & 0.89 & 117 \\
\hline TCGA-KIRC & 0.93 & 0.93 & 0.93 & 129 & 0.91 & 0.93 & 0.92 & 129 & 0.94 & 0.96 & 0.95 & 129 \\
\hline TCGA-KIRP & 0.9 & 0.88 & 0.89 & 65 & 0.93 & 0.88 & 0.9 & 65 & 0.92 & 0.88 & 0.9 & 65 \\
\hline TCGA-LAML & 1 & 0.98 & 0.99 & 53 & 0.96 & 0.98 & 0.97 & 53 & 1 & 0.98 & 0.99 & 53 \\
\hline TCGA-LGG & 0.92 & 0.97 & 0.94 & 124 & 0.98 & 1 & 0.99 & 124 & 0.98 & 0.99 & 0.99 & 124 \\
\hline TCGA-LIHC & 0.97 & 0.97 & 0.97 & 73 & 0.91 & 0.99 & 0.95 & 73 & 0.97 & 1 & 0.99 & 73 \\
\hline TCGA-LUAD & 0.88 & 0.83 & 0.85 & 126 & 0.87 & 0.81 & 0.84 & 126 & 0.9 & 0.9 & 0.9 & 126 \\
\hline TCGA-LUSC & 0.68 & 0.72 & 0.7 & 97 & 0.71 & 0.79 & 0.75 & 94 & 0.78 & 0.82 & 0.8 & 94 \\
\hline TCGA-OV & 0.99 & 0.99 & 0.99 & 73 & 0.96 & 1 & 0.98 & 73 & 1 & 0.97 & 0.99 & 73 \\
\hline TCGA-PAAD & 0.79 & 0.74 & 0.76 & 35 & 0.86 & 0.69 & 0.76 & 35 & 1 & 0.94 & 0.97 & 35 \\
\hline TCGA-PRAD & 1 & 0.99 & 0.99 & 94 & 0.99 & 0.99 & 0.99 & 94 & 1 & 0.99 & 0.99 & 94 \\
\hline TCGA-READ & 0.24 & 0.12 & 0.16 & 33 & 0.31 & 0.33 & 0.32 & 33 & 0.27 & 0.7 & 0.39 & 33 \\
\hline TCGA-STAD & 0.93 & 0.92 & 0.92 & 97 & 0.87 & 0.88 & 0.87 & 97 & 0.93 & 0.94 & 0.93 & 97 \\
\hline TCGA-THCA & 0.99 & 1 & 1 & 120 & 1 & 0.99 & 1 & 120 & 1 & 1 & 1 & 120 \\
\hline TCGA-UCEC & 0.92 & 0.91 & 0.92 & 132 & 0.93 & 0.87 & 0.9 & 132 & 0.93 & 0.96 & 0.95 & 132 \\
\hline micro avg & 0.88 & 0.88 & 0.88 & 1909 & 0.88 & 0.88 & 0.88 & 1909 & 0.9 & 0.9 & 0.9 & 1909 \\
\hline macro avg & 0.83 & 0.82 & 0.83 & 1909 & 0.85 & 0.84 & 0.85 & 1909 & 0.89 & 0.89 & 0.88 & 1909 \\
\hline weighted avg & 0.87 & 0.88 & 0.87 & 1909 & 0.88 & 0.88 & 0.88 & 1909 & 0.92 & 0.9 & 0.91 & 1909 \\
\hline
\end{tabular}

FIGURE 8 | In this figure, the first was the result of $k$-nearest neighbor $(k=5)$ algorithm, and its prediction accuracy was only $88 \%$; the second was the result of decision tree algorithm, and the classification accuracy was only $88 \%$; the third is the result of naive Bayesian algorithm, and the classification accuracy was reaching to $90 \%$.

for cancer type. That is, to calculate the Gini score $S_{j}$ for each feature $\mathrm{g}_{\text {j }}$, the calculation formula of Gini index is as follows:

$$
\mathrm{GI}_{\mathrm{m}}=1-\sum_{\mathrm{c}=1}^{|\mathrm{C}|} \mathrm{P}_{\mathrm{mk}}^{2}
$$

where $c$ represents $\mathrm{C}$ categories, and $\mathrm{P}_{\mathrm{mk}}$ represents the proportion of category $\mathrm{k}$ in node $\mathrm{m}$.
The importance of feature $g_{j}$ in node $m$, that is, the variation of Gini impurities before and after node $\mathrm{m}$ branch, is calculated as follows:

$$
\mathrm{S}_{\mathrm{jm}}=\mathrm{GI}_{\mathrm{m}}-\mathrm{GI}_{1}-\mathrm{GI}_{\mathrm{r}}
$$

where $\mathrm{GI}_{1}$ and $\mathrm{GI}_{\mathrm{r}}$, respectively, represent the Gini index of the two new nodes after branching, and $S_{j m}$ represents the importance of feature $g_{j}$ in node $\mathrm{m}$. 
If the node $m$ with characteristic $g_{j}$ that appears in decision tree $\mathrm{i}$ belongs to $\mathrm{M}$, the importance of $\mathrm{g}_{j}$ in the ith tree is calculated as follows:

$$
S_{i j}=\sum_{m \in M} S_{j m}
$$

Assuming the random forest has t trees, the importance score formula of forest is:

$$
\mathrm{S}_{\mathrm{j}}^{*}=\sum_{\mathrm{i}=1}^{\mathrm{t}} \sum_{\mathrm{m} \in \mathrm{M}} \mathrm{S}_{\mathrm{jm}}
$$

The importance score is obtained by normalizing all the importance scores obtained:

$$
S_{j}=\frac{S_{j}^{*}}{\sum_{i=1}^{n} S_{i}}
$$

The top $\mathrm{N}$ genes with high scores were selected until the stopping criterion was met. Finally, the selected genes in all samples participated in the next classification.

\section{Enrichment}

Using the gene ontology (Bindea et al., 2009; Gene Ontology Consortium, 2019) as the database of enrichment analysis and annotating the function of specific gene sets to analyze their biological significance, ClueGO (Zhao et al., 2014) is used for visualization.

\section{Classification}

In this paper, naive Bayes was used as the classifier of gene combination. Naive Bayes is one of the classical machine learning algorithms. It is a classification algorithm based on Bayes theorem. Its principle is simple and easy to implement. The core idea of naive Bayesian algorithm is to assume that each feature is independent. For a given type of data to be judged, classify and predict according to the training dataset, and calculate the probability that the current type of data to be judged belongs to a certain category through Bayesian theorem. The maximum probability relationship obtained is that the algorithm judges the category of these data. Naive Bayesian algorithm can be divided into three parts:

First, determine the feature attributes; that is to say, the expression profiles of 2,284 genes corresponding to each sample were extracted. Then, it was assumed that all the features conformed to the Gauss distribution. The samples in the dataset were labeled as cancer type. G represents the characteristics and $C$ represents the type of cancer, which can be calculated as the prior probability $\mathrm{P}(\mathrm{C}) . \mathrm{C}_{\mathrm{k}}$ represents the kth category, $\mathrm{g}_{\mathrm{i}}$ represents the ith feature, and then calculate conditional probability by prior probability. The formula is as follows:

$$
\mathrm{P}\left(\mathrm{G} \mid \mathrm{C}_{\mathrm{k}}\right)=\mathrm{P}\left(\mathrm{G}_{1}=\mathrm{g}_{1}, \mathrm{G}_{2}=\mathrm{g}_{2}, \cdots, \mathrm{G}_{\mathrm{n}}=\mathrm{g}_{\mathrm{n}} \mid \mathrm{C}_{\mathrm{k}}\right)
$$

The conditional probability of all the kth classes is calculated by the Bayesian formula:

$$
P\left(C_{k} \mid G\right)=\frac{P\left(G \mid C_{k}\right) P\left(C_{k}\right)}{P(G)}=P\left(C_{k}\right) \prod_{i=1}^{n} P\left(g_{i} \mid C_{k}\right)
$$

Since all the features conform to the Gaussian distribution and are independent of each other, the formula for conditional probability becomes as follows:

$$
\begin{aligned}
P\left(G \mid C_{k}\right) & =\prod_{i=1}^{n} P\left(g_{i} \mid C_{k}\right)=\prod_{i=1}^{n} P\left(g_{i} \mid \mu_{i, C_{k}}, \sigma_{i, C_{k}}\right) \\
& =\frac{1}{\sigma_{i, C_{k}} \sqrt{2 \pi}} \exp \left\{-\frac{\left(g_{i}-\mu_{i, C_{k}}\right)^{2}}{2 \sigma_{i, C_{k}}^{2}}\right\}
\end{aligned}
$$

where $g_{i}$ is the ith feature, and $\mu_{i, c k}$ and $\sigma_{i, c k}$ are the mean and variance of the ith feature in the $K$ class $C_{k}$, respectively.

The conditional probability formulas for all the Kth class are calculated as follows:

$$
P\left(C_{k} \mid G\right)=\frac{P\left(G \mid C_{k}\right) P\left(C_{k}\right)}{\sum_{k} P\left(G \mid C_{k}\right) P\left(C_{k}\right)} \propto P\left(G \mid C_{k}\right) P\left(C_{k}\right)
$$

Finally, obtain the relationship between the maximum probability data to be classified and the category, $\mathrm{P}\left(\mathrm{C}_{\mathrm{k}} \mid \mathrm{G}\right)$, that is:

$$
\mathrm{y}=\operatorname{argmax}_{\mathrm{C}_{\mathrm{k}}} \mathrm{P}\left(\mathrm{G} \mid \mathrm{C}_{\mathrm{k}}\right) \mathrm{P}\left(\mathrm{C}_{\mathrm{k}}\right)
$$

It is meaningful to indicate that we could get the most probable type of cancer under certain gene expressions.

\section{DISCUSSION}

In Figure 3, 20 known primary tumors were predicted, while 4 of them were misjudged, which may be related to the naive Bayesian algorithm. Naive Bayes is one of the few algorithms based on probability theory, which is a very simple and convenient algorithm. However, the premise of this algorithm is to assume that each feature is independent of others, which is not in line with the reality. Therefore, it may produce errors in the classification results, leading to the decline of the prediction accuracy. In addition, in Figure 3, COAD was mislabeled as READ. It was possibly because the anatomical proximity is relatively close and may share differential gene expression. During the normal digestive process, the function of colon and rectum is not significantly different, while colon may contribute to maintaining the gut microenvironment. The epithelial cells that are usually changed in colon adenocarcinoma and rectum adenocarcinoma are not well-distinguished. It may possibly increase both the subjective and objective bias of our model. One case of CESC was misdiagnosed as UCES. Those two female malignant tumors are more commonly regulated by the female hormone, which share similar risk factors. The anatomical proximity is close as well. The above cases indicated that anatomical proximity may share oncogenic genes to drive genetic mutation, such as both cancers contain KRAS 
mutations (Gene Ontology Consortium, 2019), or it is difficult to differentiate epithelial or adrenal cell changes before oncogenesis. It is critical to point out that some biological factors might bring some effect for model performance. It is necessary to be considered as the model construction. In addition, there are only 20 cases of known primary tumor data used to predict the classification. The data size is relatively small, so we cannot get a certain conclusion. We need to further expand the database for classification and prediction.

In Figure 4, the first 100 genes with the highest score are selected by the random forest algorithm. Some genes obtained by this method may have high correlation; that is to say, these genes will provide the same information for the classifier. In addition, although we used the 10 -fold cross-validation to investigate the performance of the model, in the independent validation, the accuracy of this model is only $75 \%$. The predictive error to PAAD is large, and the independent validation dataset is small.

\section{CONCLUSION}

In this study, the random forest and naive Bayesian algorithms were employed to trace the origin of CUP sites. Through a large number of experiments, we found that 2,284 genes with the highest score achieved the best performance. Performance evaluation shows that this method can achieve good classification and prediction results. In addition, ClueGO enrichment analysis was used for the top 100 genes with the highest scores. The results showed that some genes were enriched in PPAR signaling pathway. Upregulation of PPAR signaling pathway has been proven to lead to metabolic homeostasis disorder, inflammation, ROS accumulation, and carcinogenesis. In summary, the

\section{REFERENCES}

Anderson, G. G., and Weiss, L. M. (2010). Determining tissue of origin for metastatic cancers: meta-analysis and literature review of immunohistochemistry performance. Appl. Immunohistochem. Mol. Morphol. 18, 3-8. doi: 10.1097/pai.0b013e3181a75e6d

Ashburner, M., Ball, C. A., Blake, J. A., Botstein, D., Butler, H., Cherry, J. M., et al. (2000). Gene ontology: tool for the unification of biology. The gene ontology consortium. Nat. Genet. 25, 25-29. doi: 10.1038/75556

Bindea, G., Mlecnik, B., Hackl, H., Charoentong, P., Tosolini, M., Kirilovsky, A., et al. (2009). ClueGO: a Cytoscape plug-in to decipher functionally grouped gene ontology and pathway annotation networks. Bioinformatics 25, 1091-1093. doi: 10.1093/bioinformatics/btp101

Boscolo-Rizzo, P., Schroeder, L., Romeo, S., and Pawlita, M. (2015). The prevalence of human papillomavirus in squamous cell carcinoma of unknown primary site metastatic to neck lymph nodes: a systematic review. Clin. Exp. Metast. 32, 835-845. doi: 10.1007/s10585-015-9744-z

Brugarolas, J. (2007). Renal-cellcarcinoma: molecularpathways and therapies. N. Engl. J. Med. 356, 185-187.

Carmeliet, P., and Jain, R. K. (2011). Principles and mechanisms of vessel normalization for cancer and other angiogenic diseases. Nat. Rev. Drug Discov. 10, 417-427. doi: 10.1038/nrd3455

Chen, L. M., and Chen, B.-S. (2001). A robust adaptive DFE receiver for DSCDMA systems under multipath fading channels. IEEE Trans. Signal Process. 49, 1523-1532. doi: 10.1109/78.928705

Economopoulou, P., Mountzios, G., Pavlidis, N., and Pentheroudakis, G. (2015). Cancer of unknown primary origin in the genomic era: elucidating the dark proposed approach can reduce the cost and has high efficiency, and thus it is promising for clinical practices.

\section{DATA AVAILABILITY STATEMENT}

Publicly available datasets were analyzed in this study. This data can be found here: https://dcc.icgc.org/releases/release_26/.

\section{AUTHOR CONTRIBUTIONS}

JY, BL, and WZ conceived the project. XL and BW implemented the experiments and analyzed the data. XL, XM, and RL prepared the data and performed literature search. XL and JY wrote the manuscript. All authors approved the final manuscript.

\section{FUNDING}

This work was supported by the National Nature Science Foundation of China (Grant Nos. 11926412, 11926205, 61863010, and 61873076), the Natural Science Foundation of Hainan, China (Grant No. 119MS036), and Innovative scientific research projects for graduate students in Hainan Province (Grant No. Hys2019-266).

\section{SUPPLEMENTARY MATERIAL}

The Supplementary Material for this article can be found online at: https://www.frontiersin.org/articles/10.3389/fbioe. 2020.607126/full\#supplementary-material

box of cancer. Cancer Treat. Rev. 41, 598-604. doi: 10.1016/j.ctrv.2015 05.010

Gene Ontology Consortium (2019). The Gene ontology resource: 20 years and still going strong. Nucleic Acids Res. 47, D330-D338. doi: 10.1093/nar/gky1055

Guntinas-Lichius, O., Peter Klussmann, J., Dinsh, S., Dinh, M., Schmidt, M., Semrau, R., et al. (2006). Diagnostic work-up and outcome of cervical metastases from an unknown primary. Acta Otolaryngol. 126, 536-544. doi: 10.1080/00016480500417304

Gupta, G. P., Perk, J., Acharyya, S., de Candia, P., Mittal, V., Todorova-Manova, K., et al. (2007). ID genes mediate tumor reinitiation during breast cancer lung metastasis. Proc. Natl. Acad. Sci. U.S.A. 104, 19506-19511. doi: 10.1073/pnas. 0709185104

Hainsworth, J. D., and Greco, F. A. (2014). Gene expression profiling in patients with carcinoma of unknown primary site: from translational research to standard of care. Virchows Arch. 464, 393-402. doi: 10.1007/s00428-014-15452

Hashimoto, K., Sasajima, Y., Ando, M., Yonemori, K., Hirakawa, A., Furuta, K., et al. (2012). Immunohistochemical profile for unknown primary adenocarcinoma. PLoS One 7:e31181. doi: 10.1371/journal.pone.0031181

Hoadley, K. A., Yau, C., Wolf, D. M., Cherniack, A. D., Tamborero, D., Ng, S., et al. (2014). Multiplatform analysis of 12 cancer types reveals molecular classificationwithin and across tissues of origin. Cell 158, 929-944. doi: 10.1016/ j.cell.2014.06.049

Hudis, C. A. (2007). Trastuzumab: mechanism of action and use in clinical practice. N. Engl. J. Med. 357, 39-51. doi: 10.1056/nejmra043186

Joyce, J. A., and Pollard, J. W. (2009). Microenvironmental regulation of metastasis. Nat. Rev. Cancer 9, 239-252. doi: 10.1038/nrc2618 
Kim, K. W., Krajewski, K. M., Jagannathan, J. P., Nishino, M., Shinagare, A. B., Hornick, J. L., et al. (2013). Cancer of unknown primary sites: what radiologists need to know and what oncologists want to know. AJR Am. J. Roentgenol. 200, 484-492. doi: 10.2214/ajr.12.9363

Lv, Z., Jin, S., Ding, H., and Zou, Q. (2019). A random forest sub-Golgi protein classifier optimized via dipeptide and amino acid composition features. Front. Bioeng. Biotechnol. 7:215. doi: 10.3389/fbioe.2019.00215

Lv, Z., Zhang, J., Ding, H., and Zou, Q. (2020). RF-PseU: a random forest predictor for RNA Pseudouridine sites. Front. Bioeng. Biotechnol. 8:134. doi: 10.3389/ fbioe.2020.00134

Ma, X. J., Patel, R., Wang, X., Salunga, R., Murage, J., Desai, R., et al. (2005). Molecular classification of human cancers using a 92-gene real-time quantitative polymerase chain reaction assay. Arch. Pathol. Lab. Med. 130, $465-473$.

MacReady, N. (2010). NICE issues guidance on cancer of unknown primary. Lancet Oncol. 11:824. doi: 10.1016/s1470-2045(10)70215-1

Marquard, A. M., Birkbak, N. J., Thomas, C. E., Favero, F., Krzystanek, M., Lefebvre, C., et al. (2015). Tumor tracer: a method to identify the tissue of origin from the somatic mutations of a tumor specimen. BMC Med. Genom. 8:58. doi: 10.1186/s12920-015-0130-0

Massard, C., Loriot, Y., and Fizazi, K. (2011). Carcinomas of an unknown primary origin-diagnosis and treatment. Nat. Rev. Clin. Oncol. 8, 701-710. doi: 10.1038/ nrclinonc. 2011.158

Miller, K., Wang, M., Gralow, J., Dickler, M., Cobleigh, M., Perez, E. A., et al. (2007). Paclitaxel plus bevacizumab versus paclitaxel alone for metastatic breast cancer. N. Engl. J. Med. 357, 2666-2676. doi: 10.1056/nejmoa072113

Molina, R., Bosch, X., Auge, J. M., Filella, X., Escudero, J. M., Molina, V., et al. (2012). Utility of serum tumor markers as an aid in the differential diagnosis of patients with clinical suspicion of cancer and in patients with cancer of unknown primary site. Tumour Biol. 33, 463-474. doi: 10.1007/s13277-0110275-1

Monzon, F. A., Lyons-Weiler, M., Buturovic, L. J., Rigl, C. T., Henner, W. D., Sciulli, C., et al. (2009). Multicenter validation of a 1500-gene expression profile for identification of tumor tissue of origin. J. Clin. Oncol. 27, 2503-2508. doi: 10.1200/jco.2008.17.9762

Myung, J., Kim, K. B., Lindsten, K., Dantuma, N. P., and Crews, C. M. (2001). Lak of proteasome active site allostery as revealed by subunit-specific inhibitors. Mol. Cell 7, 411-420. doi: 10.1016/s1097-2765(01)00188-5

Oien, K. A. (2009). Pathologic evolution of unknown primary cancer. Semin. Oncol. 36, 8-37. doi: 10.1053/j.seminoncol.2008.10.009

Oien, K. A., and Dennis, J. L. (2012). Diagnostic work-up of carcinoma of unknown primary: from IHC to molecular profiling. Ann. Oncol. 23(Suppl. 10), x271$\mathrm{x} 277$.

Pappa, K. I., Choleza, M., Markaki, S., Giannikaki, E., Kyroudi, A., Vlachos, G., et al. (2006). Consistent absence of BRAF mutations in cervical and endometrial cancer despite KRAS mutation status. J. Gynecol. Oncol. 100, 596-600. doi: 10.1016/j.ygyno.2005.09.029

Pavlidis, N., and Fizazi, K. (2009). Carcinoma of unknown primary(CUP). Crit. Rev. Oncol. Hematol. 69, 271-278. doi: 10.1016/j.critrevonc.2008.09.005

Pavlidis, N., and Pentheroudakis, G. (2010). Cancer of unknown primary site: 20 questions to be answered. Ann. Oncol. 21(Suppl. 7), vii303-vii307.

Pavlidis, N., and Pentheroudakis, G. (2012). Cancer of unknown primary site. Lancet 379, 1428-1435.
Petrakis, D., Pentheroudakis, G., Voulgaris, E., and Pavlidis, N. (2013). Prognostication in cancer of unknown primary (CUP): development of a prognostic algorithm in 311 cases and review of the literature. Cancer Treat. Rev. 39, 701-708. doi: 10.1016/j.ctrv.2013.03.001

Petrushev, B., Tomuleasa, C., Susman, S., Sorișãu, O., Aldea, M., Kacsó, G., et al. (2011). The aixs of evil in the fight against cancer. Rom. J. Intern. Med. 49, 319-325.

Pillai, R., Deeter, R., Rigl, C. T., Nystrom, J. S., Miller, M. H., Buturovic, L., et al. (2011). Validation and reproducibility of a microarray-based gene expression test for tumor identification in formalin-fixed, paraffinembedded specimens. J. Mol. Diagn. 13, 48-56. doi: 10.1016/j.jmoldx.2010. 11.001

Ru, X., Li, L., and Zou, Q. (2019). Incorporating distance-based top-n-gram and random forest to identify electron transport proteins. J. Proteom. Res. 18, 2931-2939. doi: 10.1021/acs.jproteome.9b00250

Stoyianni, A., Pentheroudakis, G., and Pavlidis, N. (2011). Neuroendocrine carcinoma of unknown primary: a systematic review of the literature and a comparative study with other neuroendocrine tumors. Cancer Treat. Rev. 37, 358-365. doi: 10.1016/j.ctrv.2011.03.002

Sun, X. F., and Zhang, H. (2006). Clinicopathological significance of stromal variables: angiogenesis, lymphangiogenesis, inflammatory infiltration, MMP and PINCH in colorectal carcinomas. Mol. Cancer 5:43.

Susman, S., Tomuleasa, C., Soritau, O., Mihu, C., Rus-Ciuca, D., Sabourin, J. C., et al. (2012). The colorectal cancer stem-like cell hypothesis: a pathologist's point of view. J. BUON 17, 230-236.

Tang, W., Wan, S., Yang, Z., Teschendorff, A. E., and Zou, Q. (2018). Tumor origin detection with tissue-specific miRNA and DNA methylation markers. Bioinformatics 34, 398-406. doi: 10.1093/bioinformatics/ btx622

Tsao, M. S., Sakurada, A., Cutz, J. C., Zhu, C. Q., Kamel-Reid, S., Squire, J., et al. (2005). Erlotinib in lung cancer: molecular and clinical predictors of outcome. N. Engl. J. Med. 353, 133-144.

Varadhachary, G. R., Raber, M. N., Matamoros, A., and Abbruzzese, J. L. (2008). Carcinoma of unknown primary with a colon-cancer profile-changing paradigm and emerging definitions. Lancet Oncol. 9, 596-599. doi: 10.1016/ s1470-2045(08)70151-7

Zhao, X., Zou, Q., Liu, B., and Liu, X. (2014). Exploratory predicting protein folding model with random forest and hybrid features. Curr. Proteom. 11, 289-299. doi: $10.2174 / 157016461104150121115154$

Conflict of Interest: BW, JY, and XM were employed by company Geneis (Beijing) Co., Ltd.

The remaining authors declare that the research was conducted in the absence of any commercial or financial relationships that could be construed as a potential conflict of interest.

Copyright (C) 2020 Liang, Zhu, Liao, Wang, Yang, Mo and Li. This is an open-access article distributed under the terms of the Creative Commons Attribution License (CC BY). The use, distribution or reproduction in other forums is permitted, provided the original author(s) and the copyright owner(s) are credited and that the original publication in this journal is cited, in accordance with accepted academic practice. No use, distribution or reproduction is permitted which does not comply with these terms. 